\title{
Canonical and noncanonical Wnts use a common mechanism to activate completely unrelated coreceptors
}

\author{
Luca Grumolato, ${ }_{1}^{1}$ Guizhong Liu, ${ }^{1}$ Phyllus Mong, ${ }^{1}$ Raksha Mudbhary, ${ }^{1}$ Romi Biswas, ${ }^{1}$ \\ Randy Arroyave, ${ }^{1}$ Sapna Vijayakumar, ${ }^{1}$ Aris N. Economides, ${ }^{2}$ and Stuart A. Aaronson ${ }^{1,3}$ \\ ${ }^{1}$ Department of Oncological Sciences, Mount Sinai School of Medicine, New York, New York 10029, USA; ${ }^{2}$ Regeneron \\ Pharmaceuticals, Inc., Tarrytown, New York 10591, USA
}

\begin{abstract}
Wnt ligands signal through $\beta$-catenin and are critically involved in cell fate determination and stem/progenitor self-renewal. Wnts also signal through $\beta$-catenin-independent or noncanonical pathways that regulate crucial events during embryonic development. The mechanism of noncanonical receptor activation and how Wnts trigger canonical as opposed to noncanonical signaling have yet to be elucidated. We demonstrate here that prototype canonical Wnt3a and noncanonical Wnt5a ligands specifically trigger completely unrelated endogenous coreceptors-LRP5/6 and Ror1/2, respectively-through a common mechanism that involves their Wnt-dependent coupling to the Frizzled (Fzd) coreceptor and recruitment of shared components, including dishevelled (Dvl), axin, and glycogen synthase kinase 3 (GSK3). We identify Ror2 Ser 864 as a critical residue phosphorylated by GSK3 and required for noncanonical receptor activation by Wnt5a, analogous to the priming phosphorylation of low-density receptor-related protein 6 (LRP6) in response to Wnt3a. Furthermore, this mechanism is independent of Ror2 receptor Tyr kinase functions. Consistent with this model of Wnt receptor activation, we provide evidence that canonical and noncanonical Wnts exert reciprocal pathway inhibition at the cell surface by competition for Fzd binding. Thus, different Wnts, through their specific coupling and phosphorylation of unrelated coreceptors, activate completely distinct signaling pathways.
\end{abstract}

[Keywords: Ror1/2; LRP5/6; Wnt3a; Wnt5a; receptor activation; noncanonical Wnt signaling]

Supplemental material is available at http://www.genesdev.org.

Received June 8, 2010; revised version accepted September 20, 2010.

The Wnt family, which includes 19 members in mammals, is essential for embryonic development and tissue homeostasis (Clevers 2006; Angers and Moon 2009). Ligands such as Wnt1, Wnt3a, and Wnt8 couple the seventransmembrane domain receptor Frizzled (Fzd) and the single-membrane-spanning low-density receptor-related protein 5/6 (LRP5/6) (MacDonald et al. 2009) to activate Wnt- $\beta$-catenin signaling. In this pathway, Fzd recruits the intracellular protein dishevelled (Dvl), which in turn brings to the membrane the axin-GSK3 (glycogen synthase kinase 3) complex, thereby promoting the initial phosphorylation of LRP6 (Zeng et al. 2005, 2008). Further phosphorylation of LRP6 by casein kinase 1 (CK1) (Davidson et al. 2005; Zeng et al. 2005) is associated with clustering of different proteins-including LRP6, Dvl, and Axin-to form what has been defined as the LRP6 signalosome (Bilic et al. 2007). Phosphorylated LRP5/6 leads to inhibition of the so-called $\beta$-catenin destruction complex

${ }^{3}$ Corresponding author.

E-MAIL stuart.aaronson@mssm.edu; FAX (212) 987-2240.

Article is online at http://www.genesdev.org/cgi/doi/10.1101/gad.1957710. (which includes axin, GSK3, Dvl, CK1, and the tumor suppressor adenomatous polyposis coli), resulting in the stabilization and translocation of $\beta$-catenin in the nucleus, where it activates target genes through binding to TCF/LEF transcription factors (Clevers 2006; MacDonald et al. 2009). Among its different functions, the $\beta$-catenin or canonical Wnt pathway is a major regulator of stem/ progenitor cell maintenance, expansion, and lineage specification in both embryonic and adult tissues (Grigoryan et al. 2008). Aberrations of this pathway that lead to its constitutive activation by a variety of mechanisms-such as gene mutations, ligand overexpression, or inhibitor down-regulation-are involved in many types of cancer (Clevers 2006).

Other so-called noncanonical Wnt pathways, defined as Wnt- and/or Fzd-mediated signaling independent of $\beta$-catenin transcriptional activity (Semenov et al. 2007), are diverse and include the Wnt polarity, $\mathrm{Wnt}-\mathrm{Ca}^{2+}$, and Wnt-atypical protein kinase $\mathrm{C}$ pathways. These pathways have been reported to contribute to developmental processes such as planar cell polarity in Drosophila, convergent extension movements during gastrulation, or neuronal 
and epithelial cell migration (Semenov et al. 2007; Simons and Mlodzik 2008; Kikuchi et al. 2009). The recent identification of Wnt-binding domains in different cell membrane proteins such as Ror2 and Ryk has suggested that noncanonical family members-including Wnt4, Wnt5a, and Wnt11-may be able to trigger receptors other than Fzd (Green et al. 2008; Angers and Moon 2009). Genetic interactions between Ror2 and Wnt5a were suggested by observations that mice with inactivation of the gene encoding Ror2 showed striking similarities to $W n t 5 a^{-1-}$ mice, including perinatal lethality, dwarfism, facial abnormalities, and limb shortening (Green et al. 2008). Of note, Ror2 ${ }^{-/-}$embryos also exhibited defects in the orientation of the sensory hair cells in the inner ear, a hallmark of Wnt planar polarity pathway aberrations observed in Wnt5a $\mathrm{a}^{-/-}$, Fzd3 ${ }^{-/-}$, and Fzd6 ${ }^{-/-}$mice (Wang et al. 2006; Qian et al. 2007; Yamamoto et al. 2008). In addition, Ror2 was shown to be involved in the effects of Wnt5a in some cellular and developmental processes, such as polarized cell migration and convergent extension movements (Hikasa et al. 2002; Nishita et al. 2006; Schambony and Wedlich 2007). Recent studies have also suggested that Ror2 and Wnt5a may play a role in the progression of different types of cancers (Nishita et al. 2010a).

Unlike the case with $\beta$-catenin signaling, the mechanisms that underlie activation of noncanonical Wnt pathways are not well understood. Despite the lack of typical canonical defects in Wnt5a $a^{-/-}$mice (Yamaguchi et al. 1999; Grigoryan et al. 2008), prototype noncanonical Wnt5a has been reported to signal to $\beta$-catenin in the presence of overexpressed Fzd5 (He et al. 1997) or Fzd4 and LRP5 (Mikels and Nusse 2006). These findings argue that the pathways initiated by different Wnts may depend on the context of receptors expressed in a given target cell, rather than on intrinsic properties of the ligands (van Amerongen et al. 2008). We show that, in the same cell expressing endogenous receptors, activation of the canonical or noncanonical pathway is determined by Wnt ligand specificity for coupling Fzd to different and completely unrelated coreceptors. We further establish that prototype canonical and noncanonical Wnt3a and Wnt5a use common intracellular components-including Dvl, axin, and GSK3 - to activate LRP6 and Ror2 coreceptors, respectively, and trigger their different phenotypic responses. Finally, we provide evidence for reciprocal pathway inhibition by competition of canonical and noncanonical Wnt ligands for cell surface binding of Fzd. Given the large number of Wnts, as well as an increasing array of putative noncanonical pathways and receptors, this mechanism may represent a general paradigm underlying the activation of other, yet-to-be characterized, Wnt signaling pathways.

\section{Results}

Wnt3a and Wnt5a specifically trigger Ser/Thr phosphorylation of LRP6 and Ror2, respectively, by their coupling to a common coreceptor, Fzd

We initially compared the effects of prototype Wnt3a and Wnt5a ligands under physiological conditions in the same cells expressing endogenous canonical receptors LRP5 and LRP6 (Supplemental Fig. 1A,B), and Ror2, implicated as a noncanonical Wnt receptor (Green et al. 2008). These cells, 53S and 293T, also endogenously express Fzd4 and Fzd5 (Supplemental Fig. 1C,D; Pan et al. 2008), as Wnt5a has been reported to activate the canonical pathway in the presence of overexpressed Fzd5 (He et al. 1997) or Fzd4 and LRP5 (Mikels and Nusse 2006). Under the same conditions, Wnt $3 a$, but not Wnt5a, induced $\beta$-catenin stabilization, while both ligands triggered phosphorylation of the scaffold protein Dvl2 to similar extents, as measured by its altered gel mobility (Supplemental Fig. 2A-C), in accordance with previous reports for other cells (Gonzalez-Sancho et al. 2004). Wnt3a, but not Wnt5a, also induced phosphorylation of LRP5/6 (Fig. 1A; Supplemental Fig. 2D,E), consistent with Wnt5a's lack of canonical activity in either cell line. In contrast, Wnt5a, but not Wnt3a, induced retardation in the mobility of Ror2 (Fig. 1A; Supplemental Fig. 2D,E), which reflected its phosphorylation (Supplemental Fig. 2F; Yamamoto et al. 2007). Similar effects were observed in each of several other cell types, including mouse embryonic fibroblasts (MEFs), Saos-2, MDAMB-157, and HeLa cells (Supplemental Fig. 2G; data not shown). These results indicated that prototype canonical and noncanonical Wnts, respectively, trigger the specific phosphorylation of completely unrelated endogenous receptors.

While the mechanism involved in Wnt3a-induced phosphorylation of LRP6 is well characterized (MacDonald et al. 2009), the mechanism by which Wnt5a mediates Ror2 phosphorylation is not known. Ror2 is a receptor Tyr kinase (RTK), and there are conflicting reports that Wnt5a induces Ror2 phosphorylation on Tyr (Liu et al. 2008; Mikels et al. 2009) or Ser/Thr (Yamamoto et al. 2007) residues. In the presence of their ligands, RTKs generally are clustered and trans-autophosphorylated, which triggers the activation of downstream effectors (Schlessinger 2000). We compared the effects of treating cells expressing N-terminal Flag-tagged Ror2 with Wnt5a or anti-Flag antibody. Whereas antibody-induced Ror2 clustering triggered its Tyr autophosphorylation (Fig. 1B), Wnt5a treatment failed to do so and instead promoted Ror2 Ser/Thr phosphorylation (Fig. 1B). Of note, Wnt5ainduced Ser/Thr phosphorylation of Ror2 exhibited relatively slow kinetics (Supplemental Fig. 3A), similar to the time course of LRP6 activation by Wnt3a (Supplemental Fig. 3B; Khan et al. 2007).

It is well established that Wnt-induced coupling of LRP5/6 and Fzd coreceptors is required for canonical signaling (Tamai et al. 2000; Liu et al. 2005; Bilic et al. 2007). The fact that both Wnt $3 a$ and Wnt5a induced Dvl phosphorylation (Fig. 1A; Supplemental Fig. 2A-D; Gonzalez-Sancho et al. 2004) and increased the levels of phosphatidylinositol 4,5-bisphosphates $\left(\mathrm{PIP}_{2}\right)$ (Fig. 1C), which are hallmarks of Wnt3a-induced activation of Fzd (Pan et al. 2008; MacDonald et al. 2009), suggested that Wnt5a also possessed the inherent ability to bind and trigger Fzd. Indeed, when fused to the LRP5/6-binding domain of Dkk2, Wnt5a activated $\beta$-catenin signaling (Liu et al. 2005) and induced phosphorylation of LRP6 
A
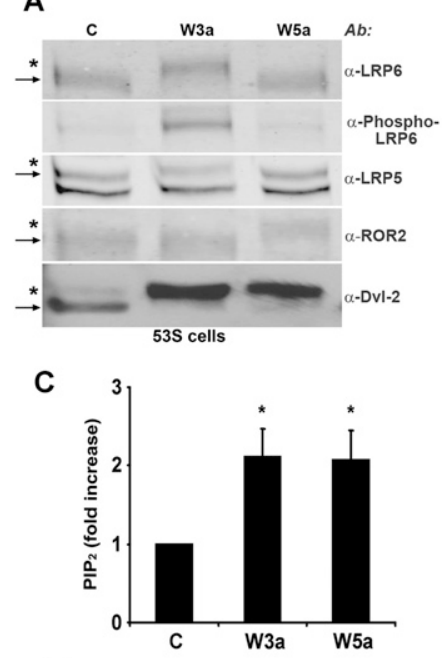

E

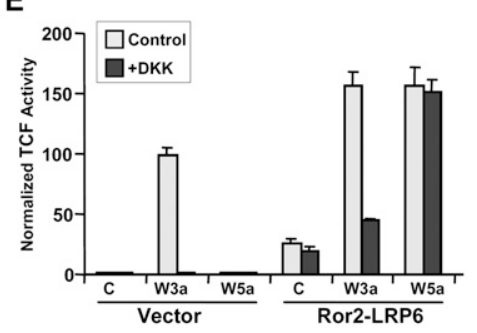

B
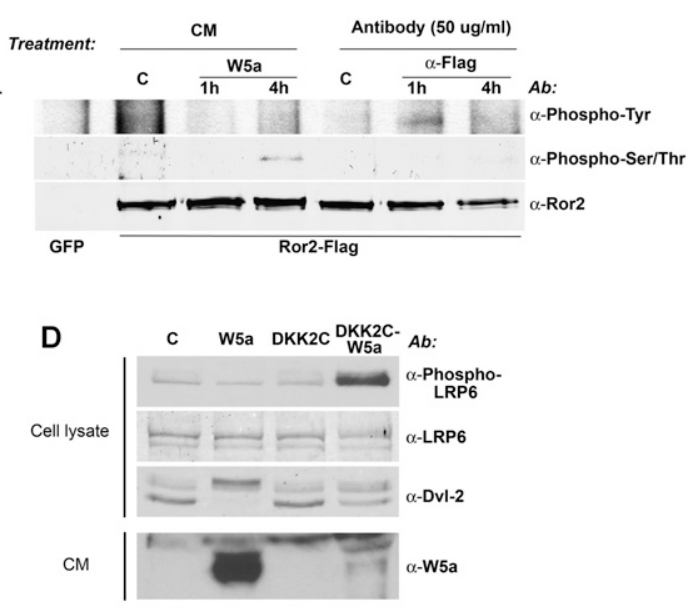

$\mathbf{F}$

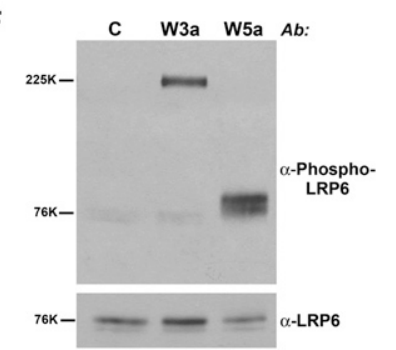

Figure 1. Prototype canonical and noncanonical Wnt ligands activate distinct endogenous receptors. (A) Opposite effects of Wnt3a and Wnt5a on LRP5/6 and Ror2 phosphorylation. $53 \mathrm{~S}$ cells were treated with control, Wnt3a, or Wnt5a CM for $3 \mathrm{~h}$, and immunoblot analysis was performed using the indicated antibodies for endogenous proteins. The nonphosphorylated forms of LRP6, LRP5, Ror2, and Dvl2 (arrow) and the upshifted bands corresponding to the phosphorylated forms (asterisk) are indicated. (B) Wnt5a induces Ror2 phosphorylation on Ser/Thr residues. Ror2 $^{-/-}$MEFs stably expressing GFP or $\mathrm{N}$-terminal Flag-tagged Ror2 were treated in the presence of the Tyr phosphatase inhibitor sodium vanadate $(40 \mu \mathrm{M})$ with either control or Wnt5a CM, or with anti-HA (control) or anti-Flag $(50 \mu \mathrm{g} / \mathrm{mL})$ monoclonal antibodies for $1 \mathrm{~h}$ or $4 \mathrm{~h}$. The cell lysates were subjected to immunoprecipitation using anti-Flag antibody, and immunoblot analysis was performed with anti-phospho-Tyr antibody, followed by two rounds of stripping and reprobing with anti-phospho-Ser/ Thr and anti-Ror2 antibodies. (C) Effects of Wnt3a and Wnt5a on PIP 2 levels. The results normalized to the control represent the mean values \pm SEM of five independent experiments. $\left(^{\star}\right) P<0.05$ compared

with control (Student's t-test). $(D)$ The DKK2C-W5a secreted fusion protein can trigger LRP6 phosphorylation. Rat1 cells were treated for $3 \mathrm{~h}$ with control, Wnt5a, DKK2C, or DKK2C-W5a CM, and immunoblot analysis was performed for endogenous LRP6 and Dvl2. The amounts of Wnt5a and DKK2C-W5a proteins in same volumes of CM are shown. $(E)$ Wnt5a stimulates the $\beta$-catenin/TCF reporter in the presence of the Ror2-LRP6 chimeric receptor. Rat1 cells were cotransfected with SuperTop luciferase reporter, renilla luciferase plasmid, and Ror2-LRP6 or control vector. Two days after transfection, the cells were treated overnight for maximal stimulation with Wnt CM in the presence or absence of Dkk1 CM, and luciferase assay was performed. The renilla-normalized values are expressed as fold increase compared with the vector-transfected control cells and correspond to the mean values $( \pm \mathrm{SD}$, in triplicate) of one representative of three independent experiments. $(F)$ Wnt5a, but not Wnt3a, induces the phosphorylation of Ror2LRP6. Two days after transfection with Ror2-LRP6, Rat1 cells were treated for $3 \mathrm{~h}$ with control, Wnt3a, or Wnt5a CM, and the cell lysates were subjected to immunoblot analysis using anti-phospho-LRP6 and anti-LRP6 antibodies. The bands close to the 225-kDa and 76-kDa markers correspond to endogenous LRP6 and Ror2-LRP6, respectively.

(Fig. 1D), indicating that the inability of Wnt5a to stimulate the $\beta$-catenin pathway was due to its lack of functional interaction with endogenous LRP5/6.

Since Wnt5a promoted Ror2 Ser/Thr phosphorylation, we speculated that Wnt5a might function by a mechanism analogous to that of canonical ligands and couple Fzd to Ror2. To explore this possibility, we generated Ror2-LRP6, a fusion receptor containing the Ror2 extracellular domain and the LRP6 transmembrane and intracellular domains. If Wnt5a triggered the coupling of the Ror2 extracellular domain to endogenous Fzd, we reasoned that we might observe phosphorylation of the LRP6 intracellular domain of this chimeric receptor and stimulation of the $\beta$-catenin pathway. Indeed, in the presence of Ror2-LRP6, Wnt5a strongly stimulated $\beta$-catenin/ TCF reporter activity (Fig. 1E). Dkk1, an antagonist of canonical signaling through interactions at the cell surface with LRP5/6 (Clevers 2006), abrogated the ability of Wnt3a, but not Wnt5a, to trigger signaling in Ror2-LRP6expressing cells (Fig. 1E). Furthermore, Wnt5a specifically induced Ser phosphorylation of Ror2-LRP6, while, under the same conditions, Wnt3a triggered phosphorylation of endogenous LRP6 (Fig. 1F). Thus, by replacing the Ror2 intracellular domain with that of LRP6, we converted Ror2 into a canonical Wnt receptor specifically activated by Wnt5a.

We next investigated the role of endogenous Fzd in Wnt5a-induced triggering of Ror2. The protein Shisa, which specifically inhibits Fzd translocation to the membrane by retaining it in the endoplasmic reticulum (Yamamoto et al. 2005), has been used to demonstrate the involvement of Fzd in Wnt3a-mediated phosphorylation of LRP6 (Zeng et al. 2008). Figure 2A confirms Shisa's inhibition of $\beta$-catenin/TCF reporter activity triggered by Wnt3a, and shows further that Shisa antagonized the ability of Wnt5a to activate Ror2-LRP6. Moreover, Shisa abrogated both the phosphorylation of Ror2 induced by Wnt5a (Fig. 2B,C) and Wnt3a-mediated activation of LRP6 (Fig. 2B). As a control for Fzd inhibition, Shisa expression blocked the upshift of Dvl2 induced by both 
A

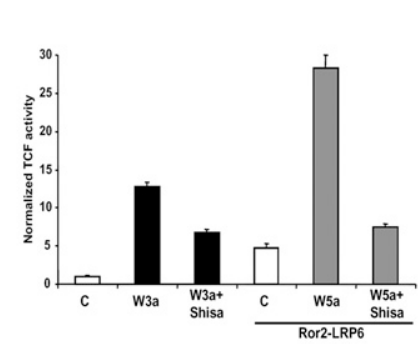

C

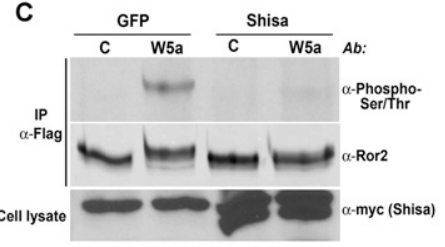

B

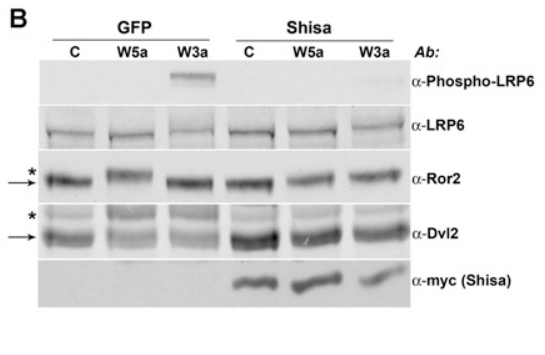

D

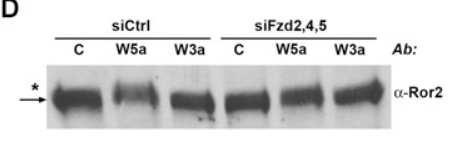

E F

G

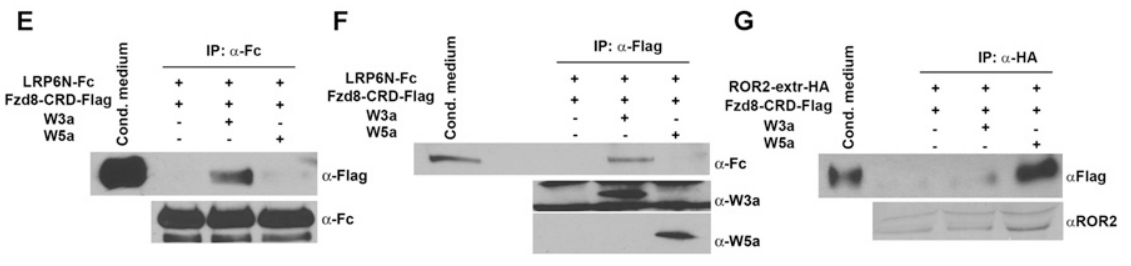

Figure 2. Wnt3a and Wnt5a couple Fzd to LRP6 or Ror2 coreceptors, respectively, to induce their phosphorylation. (A) Rat1 cells stably expressing GFP or myc-tagged Shisa were cotransfected with SuperTop luciferase reporter, renilla luciferase plasmid, and Ror2-LRP6 or control vector. Two days after transfection, the cells were treated overnight with control, Wnt $3 \mathrm{a}$, or Wnt5a $\mathrm{CM}$, and a luciferase assay was performed. The renilla-normalized values are expressed as fold increase compared with the vectortransfected control cells and correspond to the mean values $( \pm \mathrm{SD}, n=4)$ of one representative of two independent experiments. (B) Saos-2 cells stably expressing GFP or myc-tagged Shisa were treated for 3 h with control, Wnt3a, or Wnt5a CM, and immunoblot analysis was performed with the indicated antibodies. The nonphosphorylated (arrow) and phosphorylated (asterisk) Ror2 and Dvl2 are indicated. $(C)$ Ror $^{-/-}$MEFs stably expressing Flag-tagged Ror2 were transduced with GFP or Shisa lentiviruses and treated with control or Wnt5a CM overnight, followed by immunoprecipitation using anti-Flag antibody. Immunoblot analysis was performed using the indicated antibodies. $(D)$ Two days after transfection with control or Fzd2,4,5 siRNAs, 293T cells were treated for $3 \mathrm{~h}$ with Wnt3a or Wnt5a CM. Immunoblot analysis was performed using anti-Ror2 antibody. $(E-G)$ Wnt3a and Wnt5a couple Fzd to LRP6 or Ror2, respectively. CM containing receptor extracellular domains (LRP6N-Fc, Fzd8CRD-Flag, and Ror2-extr-HA) or Wnt ligands were mixed as indicated, and immunoprecipitation was performed with anti-Fc (E), anti-Flag $(F)$, or anti-HA $(G)$ antibodies, followed by immunoblot analysis using the indicated antibodies.

Wnt3a and Wnt5a (Fig. 2B). Despite the fact that there are $10 \mathrm{Fzd}$ genes encoded within the mammalian genome (MacDonald et al. 2009), siRNAs targeting Fzd2, Fzd4, and Fzd5 were shown recently to partially inhibit Wnt3ainduced phosphorylation of LRP6 in 293T cells (Pan et al. 2008). Figure 2D and Supplemental Figure 4A show that these same siRNAs also inhibited the phosphorylation of Ror2 induced by Wnt5a in these cells. Together, these results indicated that Fzd is required for the activation of both canonical and noncanonical Wnt coreceptors.

Previous studies have shown that Wnt3a directly coupled Fzd and LRP6 in an in vitro binding assay using the secreted extracellular domains of these receptors (Tamai et al. 2000). Figure 2, E and F shows that Wnt3a, but not Wnt5a, was able to bridge LRP6 and Fzd. Conversely, Wnt5a, but not Wnt3a, formed a tripartite complex with Ror2 and Fzd (Fig. 2G). All of these findings demonstrated that Wnt3a and Wnt5a physically couple Fzd, respectively, to two different and completely unrelated coreceptors.

Wnt-induced phosphorylation of LRP6 and Ror2 is mediated by a common intracellular machinery

Our findings that Wnt5a mediated coupling of Fzd to Ror2 and promoted activation of Ror2-LRP6 suggested that this ligand might induce Ser/Thr phosphorylation of Ror2 through a mechanism analogous to Wnt3a triggering of LRP6, which involves intracellular components Dvl, axin, and GSK3. Figure 3A shows that Wnt5a treat- ment promoted colocalization of aggregates staining for Ror2 and Dvl2 at the cell membrane. These Ror2 membrane aggregates were not detected in response to Ror2 shRNA (Supplemental Fig. 5). Consistent with a role of Dvl in the activation of both canonical and noncanonical receptors, down-regulation of the three Dvl isoforms by lentiviral shRNAs or expression of a dominant-negative Dvl construct (DN-Dvl), which binds to Fzd but is unable to induce phosphorylation of LRP6 (Zeng et al. 2008), inhibited the triggering of Ror2, LRP6, or Ror2-LRP6 induced by their respective ligands (Fig. 3B; Supplemental Fig. 4B,C).

In the canonical Wnt pathway, axin functions as a component of the $\beta$-catenin destruction complex as well as a scaffold protein involved in the recruitment of GSK3 to the Fzd-LRP6 complex, required for LRP6 activation by phosphorylation (Zeng et al. 2008; MacDonald et al. 2009). Figure 3C and Supplemental Figure 6A show that shRNA-mediated down-regulation of axin inhibited the phosphorylation of Ror2 or LRP6 specifically induced by Wnt5a and Wnt3a, respectively. Moreover, Wnt5a, but not Wnt3a, induced the formation of a complex containing endogenous Ror2 and axin (Fig. 3D), suggesting that Wnt5a triggered recruitment of axin-GSK3 to the FzdRor2 complex to promote Ror2 phosphorylation, similarly to what has been described for LRP6 (Zeng et al. 2008). In fact, a recent study reported that GSK3 was involved in Ror2 phosphorylation (Yamamoto et al. 2007). We confirmed these data and showed that different 
A
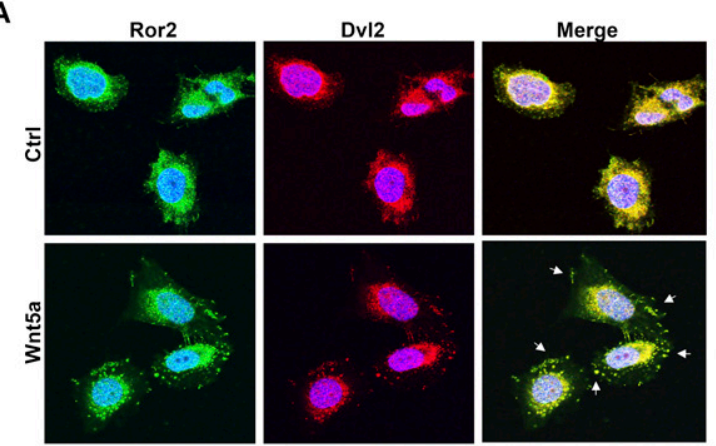

B
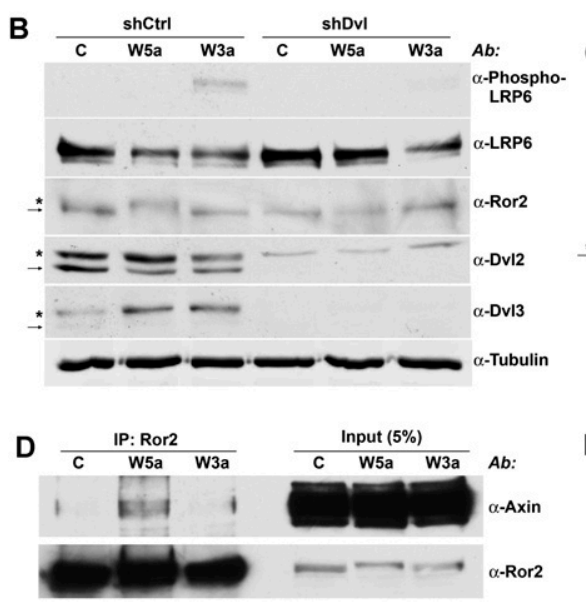

F

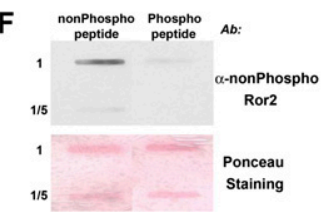

H

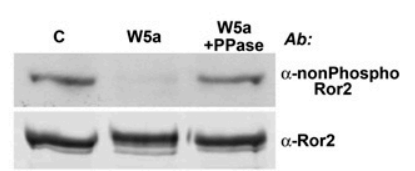

C

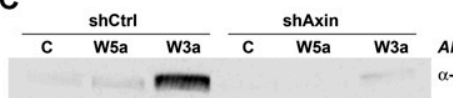
Ab:
$\alpha-P h o s$
LRPG LRP6

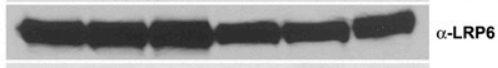

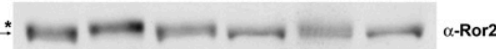

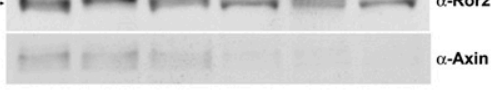

E $\frac{\text { Ror2 }}{C \quad \text { W5a }} \frac{\text { Ror2-S772A }}{C \text { W5a }}$ Ror2-S864A

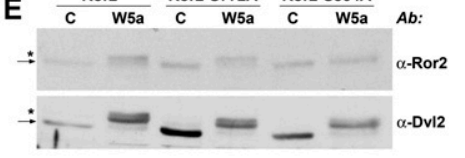

G

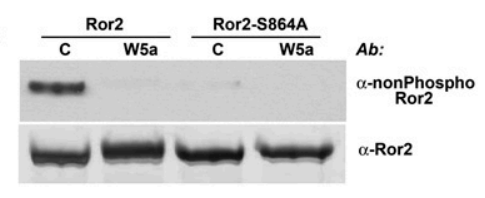

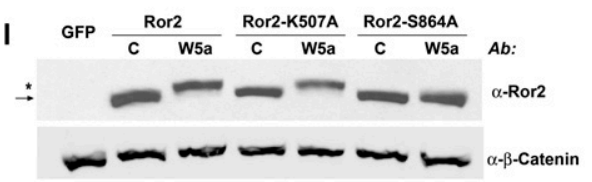

Figure 3. The same intracellular machinery is used for Wnt-induced phosphorylation of LRP6 and Ror2. (A) Colocalization of endogenous Ror2 (green) and Dvl2 (red) upon Wnt5a treatment $(2 \mathrm{~h})$ in Saos- 2 cells. (B) Dvl proteins are required for LRP6 and Ror2 phosphorylation. Saos-2 cells were transduced with shRNA lentiviral vectors targeting the three Dvl genes or a control vector and treated for $2 \mathrm{~h}$ with Wnt CM, followed by immunoblot analysis. Nonphosphorylated (arrow) and phosphorylated (asterisk) Ror2 and Dvl2,3 are indicated. $(C)$ Axin is involved in ligand-induced triggering of both Ror2 and LRP6. Two days after transduction with control or shAxin lentiviruses, Saos-2 cells were treated for $3 \mathrm{~h}$ with control, Wnt3a, or Wnt5a CM followed by immunoblot analysis. (D) Wnt5a induces formation of a complex including endogenous Ror2 and axin. MDA-MB157 cells were treated for $90 \mathrm{~min}$ with CM, followed by Ror2 immunoprecipitation and immunoblot analysis with anti-axin and anti-Ror2 antibodies. (E) Effects of putative GSK3 phosphorylation site mutations on Ror2 upshift. Two days following transfection with the indicated Ror2 constructs, Rat1 cells were treated with control or Wnt5a CM for $2 \mathrm{~h}$, followed by immunoblot analysis. (F) A slot blot generated using serial dilutions of Ror2 nonphosphopeptide and phosphopeptide was stained with Ponceau solution (loading control), followed by immunoblot analysis with anti-non-PhosphoRor2 antibody. (G) Ror2 Re $^{-/}$MEFs stably expressing Flag-tagged wild-type (wt) or S864A Ror2 constructs were treated with control or Wnt5a CM, followed by immunoprecipitation with anti-Flag antibody and immunoblot analysis. $(H)$ Ror $2^{-/-}$MEFs stably expressing Flag-tagged Ror2 were treated with control or Wnt5a CM followed by immunoprecipitation with anti-Flag antibody. The immunoprecipitates were treated in the presence or absence of $\lambda$ protein phosphatase, and immunoblot analysis was performed. (I) Ror2 Tyr kinase activity is not required for Wnt5a-induced Ror2 phosphorylation. Ror2 ${ }^{-/-}$MEFs stably expressing Flag-tagged Ror2 constructs were treated with control or Wnt5a CM, and immunoblot analysis was performed using anti-Ror2 and anti- $\beta$-catenin (loading control) antibodies.

GSK3-specific inhibitors, SB216763 and BIO, each dramatically decreased the ability of Wnt3a and Wnt5a to specifically trigger phosphorylation of LRP6 or Ror2, respectively, under the same conditions in Saos-2 cells (Supplemental Fig. 6B,C). All of these findings strongly supported a model in which the binding of Fzd to either canonical or noncanonical coreceptors mediated by Wnt ligands specific for each coreceptor leads to their phosphorylation by the same intracellular machinery.

Wnt5a-induced phosphorylation of Ror2 on Ser 864 is required for noncanonical coreceptor activity

It has been shown that GSK3 phosphorylation serves as a priming event, followed by CK1 phosphorylation, in the activation of LRP6 (MacDonald et al. 2009). We used a bioinformatics approach to analyze the Ror2 intracellular domain and found various GSK3 and CK1 putative phosphorylation sites conserved in vertebrates (Supplemental Fig. 7A). We individually substituted alanine for the two Ser residues that showed the highest scores as GSK3 sites and exogenously expressed wild-type Ror2 or these mutants in ratl cells, which lack detectable endogenous Ror2 expression (data not shown). Figure 3E shows that the Ror2-S864A mutant failed to exhibit the Wnt5ainduced upshift indicative of phosphorylation, while the S772A mutant showed the characteristic upshift. The S864A mutation also inhibited Ror2 in vitro phosphorylation by GSK3 (Supplemental Fig. 7B). Furthermore, 
a peptide antibody that specifically recognized only the nonphosphorylated Ror2 Ser 864 (Fig. 3F) detected wildtype Ror2 in the absence of Wnt5a treatment, but failed to recognize Wnt5a-triggered wild-type Ror2 or the S864A mutant receptor expressed at similar levels (Fig. 3G). Of note, the ability of this non-Phospho-Ror2 antibody to detect Wnt5a-triggered Ror2 was restored by treating the cell lysate with $\lambda$ protein phosphatase (Fig. $3 \mathrm{H}$ ). Together, these results demonstrate that Wnt5a promotes the phosphorylation of Ror2 on Ser 864. Of note, a kinasedead Ror2 construct containing a mutation in the ATPbinding domain was phosphorylated similarly to wildtype Ror2 in response to Wnt5a, while, under the same conditions, the upshift of Ror2-S864A was strongly impaired (Fig. 3I), confirming that Ror2 Tyr kinase activity is not required for the triggering of this receptor by Wnt5a.

Wnt5a has been reported to stimulate polarized cell migration by a mechanism involving Ror2 and Dvl (Nishita et al. 2006; Schlessinger et al. 2007; Nomachi et al. 2008). Figure 4A shows that Wnt5a stimulated the migration of rat1 cells containing wild-type Ror2 but not the same cells expressing Ror2-S864A at comparable levels. The kinase-dead Ror2 behaved similarly to the wild-type receptor (Supplemental Fig. 8A; Nishita et al. 2006), indicating that Ror2 Tyr kinase activity is dispensable for this function. Conversely, Ror2 mutants with S864D or S864E substitutions, which can mimic phosphorylated residues, increased ratl cell motility even in the absence of Wnt5a treatment (Fig. 4B). Consistent with these findings, exogenous expression of wild-type Ror2 in MEFs derived from mice with inactivation of the Ror2 gene (DeChiara et al. 2000) partially rescued their impaired cell motility, while Ror2-S864A had no effect (Supplemental Fig. S8B,C). These results provide biological evidence that Wnt-induced phosphorylation of Ror2 is required for activation of noncanonical signaling functions involved in cell motility.

\section{Identification of Ror1 as another noncanonical receptor specifically phosphorylated in the presence of Wnt5a}

Wnt5a has been reported to bind Ror1 in vitro (Fukuda et al. 2008). Our in vivo analysis revealed that Wnt5a, but not Wnt3a, provoked an upshift of endogenous Rorl and stimulated its phosphorylation on Ser/Thr residues in HCC1500 and HCC3153 breast cancer cells (Fig. 5A,B). Moreover, cotreatment with BIO or SB216763 inhibited both the upshift of Ror1 specifically induced by Wnt5a and LRP6 phosphorylation induced by Wnt3a (Fig. 5C; Supplemental Fig. 91, strongly suggesting that the mechanism involved in Wnt5a activation of Ror2 applied to Rorl as well. To confirm this conclusion, we generated a Ror1-LRP6 chimeric receptor containing the Ror1 extracellular domain with LRP6 transmembrane and cytoplasmic domains. Figure 5D shows that Wnt5a stimulated $\beta$-catenin/TCF reporter activity in cells expressing Ror1LRP6, but not vector control cells, indicating that this prototype noncanonical Wnt ligand functionally coupled the Rorl moiety of the chimeric receptor to endogenous Fzd and connected the chimeric receptor to canonical signaling. Furthermore, Wnt5a specifically induced phosphorylation of Ror1-LRP6, while Wnt3a instead promoted
A
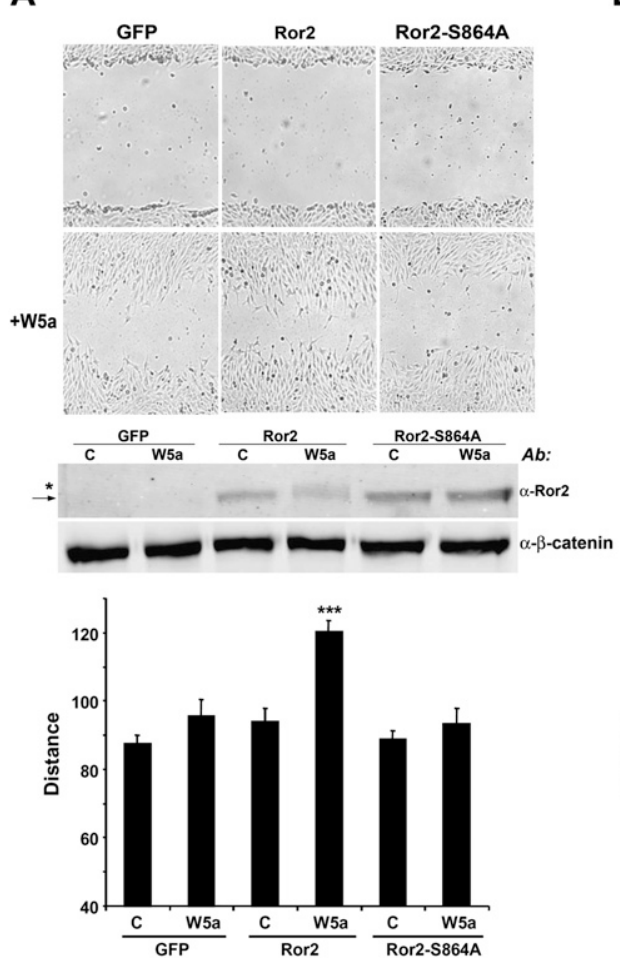

B
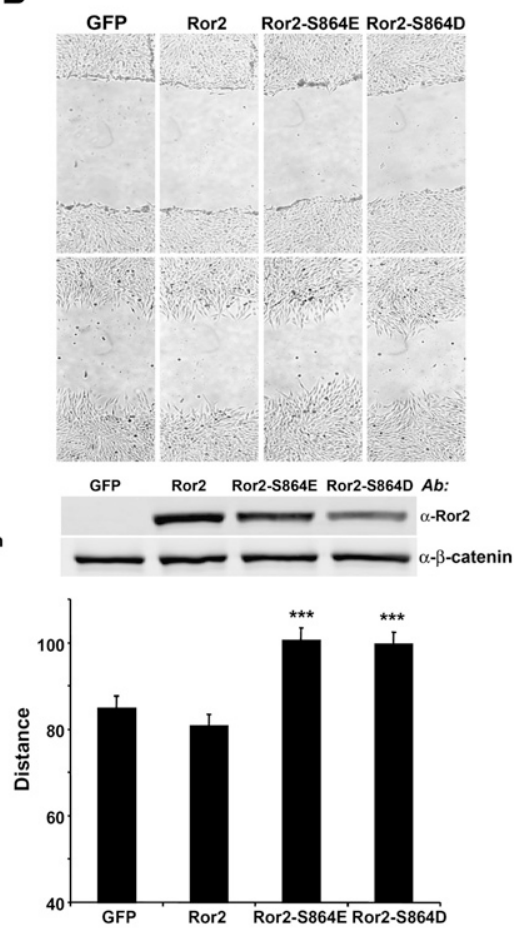

Figure 4. Ror2 phosphorylation is required for Wnt5a-induced cell migration. (A) Confluent ratl cells stably expressing GFP, Ror2, or Ror2-S864A were wounded and treated with control or Wnt5a CM $(0.5 \%$ of fetal bovine serum [FBS]), and the relative migration of the wound edge was measured after $15 \mathrm{~h}$. Mean values \pm SEM $(n=18)$ from one representative of five independent experiments are shown. (B) Rat1 cells stably expressing GFP, Ror2, Ror2-S864E, or Ror2-S864D were wounded and incubated in $0.5 \%$ FBS medium, and the relative migration was measured after $14 \mathrm{~h}$. Mean values $\pm \operatorname{SEM}(n=22)$ from one representative of three independent experiments are shown. $\left(^{\star \star \star}\right) P<0.001$ compared with all other conditions $(A)$ or with GFP-expressing cells $(B)$ (Student's $t$-test). Immunoblot analysis was performed to assess the expression levels of Ror2 constructs. 


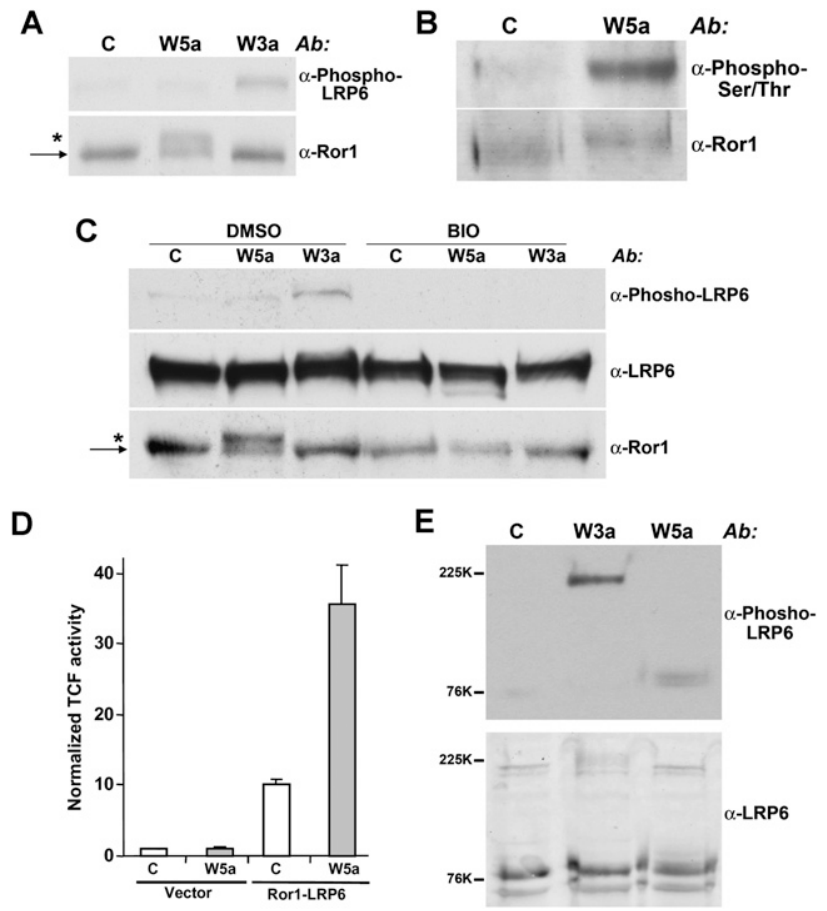

Figure 5. Rorl is specifically phosphorylated by Wnt5a. (A) HCC1500 cells were treated for $3 \mathrm{~h}$ with control, Wnt3a, or Wnt5a CM, followed by immunoblot analysis. The nonphosphorylated (arrow) and phosphorylated (asterisk) Rorl is indicated. (B) HCC3153 cells were treated for $3 \mathrm{~h}$ with control or Wnt5a CM, followed by immunoprecipitation with anti-Ror1 antibody and immunoblot analysis. (C) HCC1500 cells were pretreated for $1 \mathrm{~h}$ with or without the GSK3-specific inhibitor BIO $(20 \mu \mathrm{M})$, followed by $2 \mathrm{~h}$ treatment with control, Wnt3a, or Wnt5a CM in the presence or absence of BIO $(20 \mu \mathrm{M})$. Immunoblot analysis was performed with the indicated antibodies. $(D)$ Rat1 cells cotransfected with SuperTop luciferase reporter, renilla luciferase plasmid, and Ror1-LRP6 or control vector were treated overnight with control or Wnt5a CM, and a luciferase assay was performed. The renilla-normalized values are expressed as fold increase compared with the vector-transfected control cells and correspond to the mean values $( \pm S D, n=4)$ of one representative of three independent experiments. $(E)$ Rat 1 cells transfected with Ror1-LRP6 were treated for $3 \mathrm{~h}$ with control, Wnt3a, or Wnt5a CM, and immunoblot analysis was performed. The bands close to the $225-\mathrm{kDa}$ and $76-\mathrm{kDa}$ markers correspond to endogenous LRP6 and Ror1-LRP6, respectively.

the phosphorylation of endogenous LRP6 (Fig. 5E). Together, these results establish that Wnt5a activates Ror1 by the same mechanism responsible for Wnt3a activation of canonical signaling through LRP5/6 and Wnt5a triggered noncanonical signaling through Ror2.

\section{Cross-inhibition at the cell membrane between canonical and noncanonical Wnts}

Recent studies using exogenous Ror2 overexpression indicated that Wnt5a inhibits the canonical Wnt pathway downstream from $\beta$-catenin by a mechanism involving signaling through Ror2 (Mikels and Nusse 2006; Mikels et al. 2009). We found that neither Rorl/2 shRNA- mediated knockdown (Fig. 6A) nor Ror2 knockout (Supplemental Fig. 10A) affected the ability of Wnt5a to inhibit Wnt3a-induced $\beta$-catenin/TCF reporter activity, indicating that endogenous Ror $1 / 2$ are not strictly required for inhibition of the $\beta$-catenin pathway by Wnt5a. Based on our evidence that canonical and noncanonical Wnt receptor activation involves shared use of Fzd, we reasoned instead that an excess of either type of Wnt might interfere with the other pathway as a result of ligand competition for binding to Fzd. Consistent with this hypothesis, Wnt5a antagonized Wnt3a interaction with Fzd in an in vitro binding assay (Supplemental Fig. 10B). Moreover, Wnt5a antagonized both LRP6 phosphorylation and $\beta$-catenin stabilization induced by Wnt3a (Fig. 6B) and mimicked the effects of Dkk1 in decreasing $\beta$-catenin/TCF reporter activity in tumor cells with Wnt autocrine pathway activation, but not in those containing a $\beta$-catenin-activating mutation (Fig. 6C). Reciprocally, Figure 6, D and E, and Supplemental Figure 10C show that cotreatment with Wnt3a inhibited the ability of Wnt5a to trigger phosphorylation of both endogenous and exogenous Ror2, as assessed by analysis of Ror2 upshift or anti-phospho-Ser/Thr antibody. All of these findings demonstrate that canonical and noncanonical Wnts cross-compete for activation of the reciprocal pathway at the cell surface.

Human mesenchymal stem cells (hMSCs) can give rise in vitro and in vivo to different lineages, including osteoblasts, chondrocytes, myocytes, and adipocytes (Pittenger et al. 1999). We showed that canonical Wnts maintain hMSCs in a proliferative and undifferentiated state, while Wnt5a promotes their osteogenic differentiation (Liu et al. 2009). In fact, decreased Ror2 expression inhibited differentiation of these cells (Supplemental Fig. 11A; Liu et al. 2007) in accordance with a role of this receptor in osteogenic differentiation (Green et al. 2008). Cocultivation of hMSCs with vector control or Ror2-LRP6-expressing reporter cells revealed evidence of constitutive noncanonical Wnt ligand activity in hMSCs (Fig. 7A). To investigate whether inhibition of osteogenic differentiation induced by high levels of Wnt3a might be caused in part by canonical Wnt ligand interference with endogenous Ror2 signaling at the receptor level, we inhibited the canonical pathway downstream from LRP5/6 by expression of either lentiviral $\beta$-catenin shRNA or dominantnegative TCF4 (DN-TCF4). Despite the resulting abrogation of Wnt3a-induced $\beta$-catenin/TCF reporter activity (Fig. 7B; Supplemental Fig. 11C), we observed no impairment in the ability of Wnt3a to decrease hMSC differentiation (Fig. 7C; Supplemental Fig. 11B). These results imply that Wnt3a can inhibit osteogenic differentiation by directly antagonizing Wnt5a/Ror2 signaling at the receptor level, further supporting the concept of crosscompetition at the cell surface between canonical and noncanonical Wnts.

\section{Discussion}

Our present studies establish a novel mechanism by which distinct classes of Wnt ligands activate very 

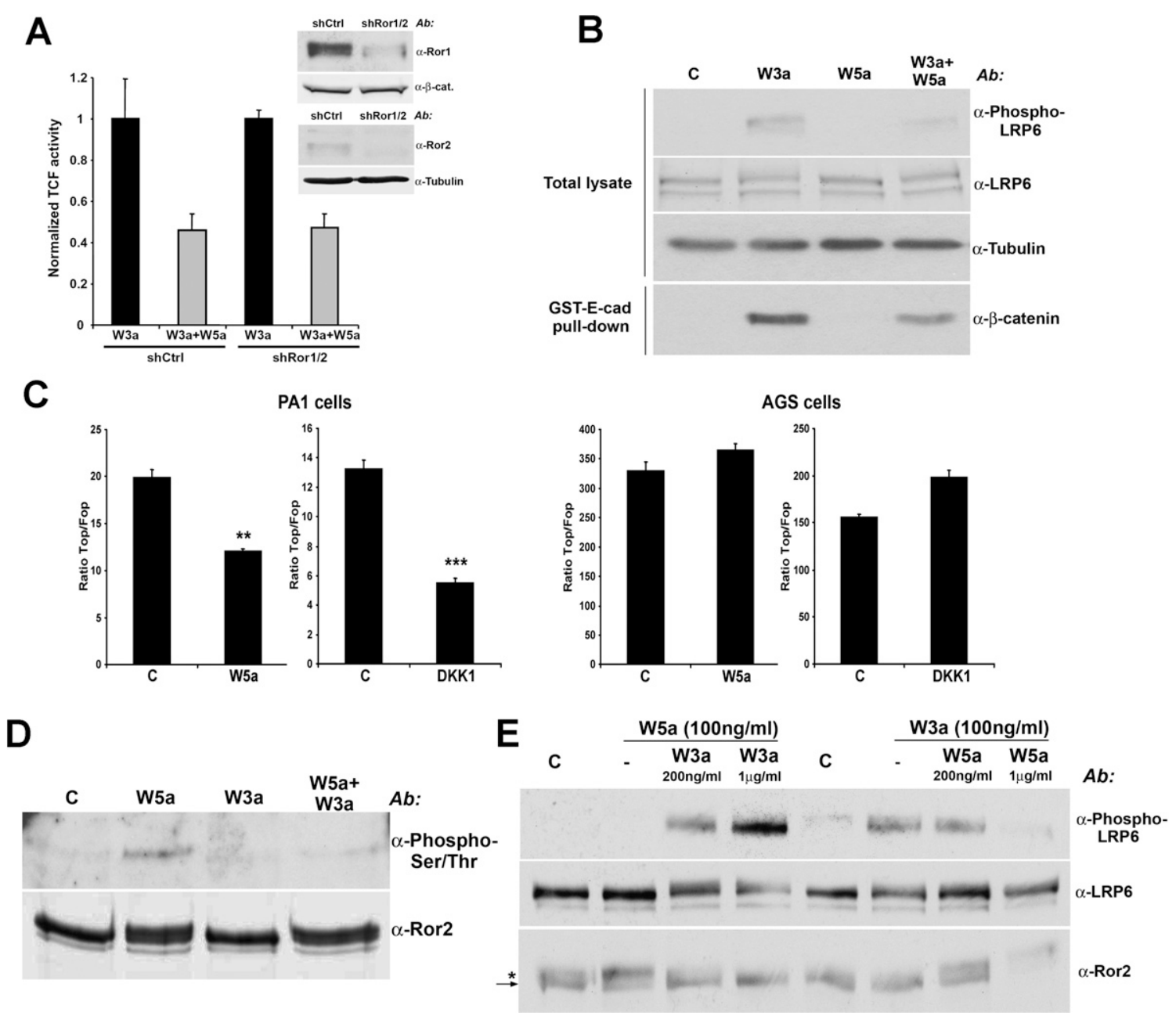

Figure 6. Cross-competition at the cell surface between canonical and noncanonical Wnt ligands. (A) 293T cells stably expressing control or Ror1/2 shRNAs and transfected with SuperTop luciferase reporter and renilla luciferase plasmid were treated overnight with Wnt3a in the presence or the absence of Wnt5a CM in a 1-to-2 ratio, and a luciferase assay was performed. The renilla-normalized values are expressed as fold decrease compared with the Wnt3a-treated cells and correspond to the mean values $( \pm \mathrm{SD}, n=4)$ of one representative of two independent experiments. Ror1/2 knockdown was assessed by immunoblot analysis. $(B)$ Wnt5a inhibits Wnt3ainduced phosphorylation of LRP6 and $\beta$-catenin stabilization. Wnt5a 293T cells were treated for $3 \mathrm{~h}$ with Wnt3a and/or Wnt5a CM in a 1-to-2 ratio, and the cell lysates were subjected to immunoblot analysis or GST-E-cadherin pull-down for analysis of uncomplexed $\beta$-catenin. (C) PAl ovarian teratocarcinoma cells (autocrine Wnt signaling) and AGS gastric adenocarcinoma cells (activating $\beta$-catenin mutation) were transduced with lentiviral mTOP- or mFOP-luciferase reporters together with a PGK-driven renilla luciferase virus and treated for $22 \mathrm{~h}$ with Wnt5a, Dkk1, or their corresponding control CM. The reporter activity was calculated by dividing the mTOP/ renilla ratio by the $\mathrm{mFOP} /$ renilla ratio, and the mean values ( \pm SEM, in triplicate) of one representative of two independent experiments are shown. $\left.\left(^{\star \star}\right) P<0.01{ }^{\star \star \star \star}\right) P<0.001$ compared with control (Student's $t$-test). $(D, E)$ Wnt3a inhibits Wnt5a-induced phosphorylation of Ror2. (D) Ror2 ${ }^{-1-}$ MEFs stably expressing Flag-tagged Ror2 were treated with Wnt5a in the presence or absence of Wnt3a CM in a 1-to-2 ratio, followed by immunoprecipitation with anti-Flag antibody and immunoblot analysis. (E) Saos2 cells were treated with the indicated amounts of purified Wnt5a and Wnt3a for $2 \mathrm{~h}$, followed by immunoblot analysis for endogenous Ror2 and LRP6. Nonphosphorylated (arrow) and phosphorylated (asterisk) Ror2 are indicated.

different signaling pathways determined by their specific abilities to couple a common receptor, Fzd, with completely unrelated endogenous coreceptors. Moreover, we established that the mechanism of activation of these unrelated receptors involves their Ser/Thr phosphorylation by shared intracellular components (Fig. 8). As one demonstration of this model, we were able to convert Wnt5a from a noncanonical to a canonical ligand by switching the extracellular domain of LRP6 with Ror1 or Ror2 external domains. This allowed Wnt5a, but not Wnt3a, to specifically induce Ser/Thr phosphorylation of the LRP6 intracellular domain of the Ror/LRP6 chimeric receptor and consequently activate the canonical pathway.

Previous studies involving overexpression of cotransfected Wnt ligands and Ror2 led to evidence that Ror2 could bind various Wnts, including canonical Wnt1, Wnt3a, and Wnt8 (Hikasa et al. 2002; Billiard et al. 2005). In contrast, in vitro analysis indicated that this 
A

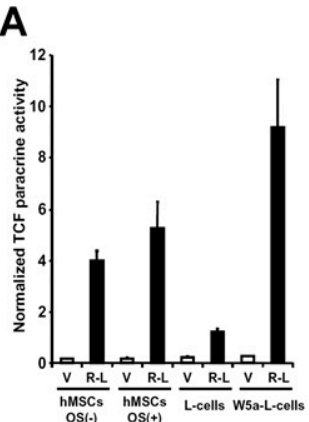

B
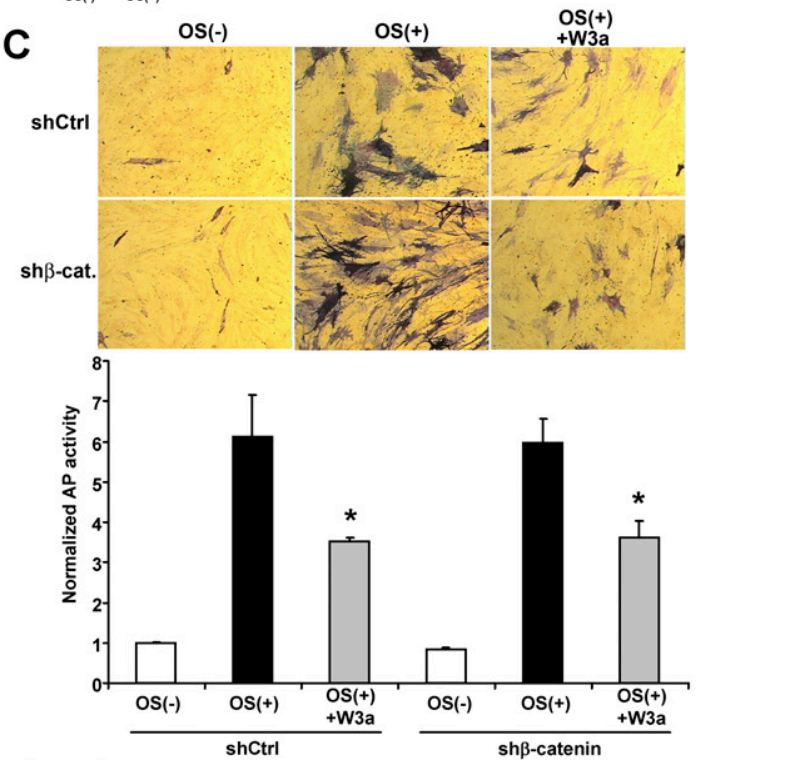

Figure 7. $\beta$-Catenin is dispensable for Wnt3a-mediated inhibition of hMSC osteogenic differentiation. $(A)$ hMSCs display endogenous noncanonical activity. Ratl cells were cotransfected with vector $(\mathrm{V})$ or Ror2-LRP6 (R-L) together with SuperTop or SuperFop and renilla luciferase plasmids. One day after transfection, reporter Ratl cells were transferred to plates containing undifferentiated or differentiated ( $4 \mathrm{~d}$ in OS medium) hMSCs, parental L cells (negative control), or Wnt5a-expressing $\mathrm{L}$ cells (positive control) for an additional day before the luciferase assay. Paracrine Ror2-LRP6 mean activities ( \pm SD) from one representative of two experiments are expressed as a ratio between Top and Fop reporters, both normalized with the renilla luciferase values. $(B)$ hMSCs containing the mTOP- or mFOP-luciferase reporters were transduced with a lentiviral shRNA vector targeting $\beta$-catenin or control vector and were treated overnight with or without purified Wnt3a $(200 \mathrm{ng} / \mathrm{mL})$ in OS medium. The reporter activity was calculated by dividing the $\mathrm{mTOP} /$ renilla ratio by the $\mathrm{mFOP} /$ renilla ratio, and the mean values ( \pm SEM,$n=4)$ of one representative of two independent experiments are represented. $\beta$-Catenin down-regulation was assessed by immunoblot analysis. $(C)$ hMSCs transduced with control or $\beta$-catenin shRNA lentiviruses were grown in normal medium or OS differentiation medium with or without purified Wnt3a (200 ng/mL). hMSC osteogenic differentiation was assessed for alkaline phosphatase activity by staining (top panel, $10 \mathrm{~d}$ of differentiation) or using a luminescent substrate (bottom panel, $6 \mathrm{~d}$ of differentiation). The results represent the mean values ( \pm SEM, $n=5$ ) of one representative of three independent experiments. $\left(^{\star}\right) P<0.05$ compared with hMSCs differentiated in the absence of Wnt3a (Student's $t$-test). receptor specifically interacted only with noncanonical Wnt5a (Oishi et al. 2003; Mikels and Nusse 2006). We demonstrated here that Wnt5a, but not Wnt3a, physically bridged Ror2 to Fzd extracellular domains. Conversely, under the same conditions, Wnt3a, but not Wnt5a, coupled Fzd to LRP6. While LRP5/6 coreceptors bind canonical Wnts through their large extracellular $\beta$-propeller domains (Clevers 2006; MacDonald et al. 2009), both Fzd and Ror1/2 bind Wnt by means of their cysteine-rich domains (CRDs) (Logan and Nusse 2004; Green et al. 2008). This 120- to 125 -amino-acid domain is present in a variety of proteins, including Fzd and Smoothened seven-transmembrane domain receptors, Ror $1 / 2$ and the muscle-specific kinase (MuSK) RTKs, and the secreted Fzd-related proteins (FRPs) (Green et al. 2008). Previous biochemical and crystallographic studies have suggested that the CRDs of Fzd and FRP could interact to form dimers (Bafico et al. 1999; Dann et al. 2001). In this regard, it has been reported that cotransfected Ror2 and Fzd coimmunoprecipitated in the absence (Oishi et al. 2003) or presence of Wnt5a (Nishita et al. 2010b). Under stringent in vitro binding conditions, we showed that Wnt5a is required for the formation of a complex containing the Fzd8 CRD and the Ror2 extracellular domain. These findings are consistent with predictions from the crystal structure of Fzd and FRP CRDs that a ligand is likely required to increase the otherwise weak dimerization affinity of this type of domain (Dann et al. 2001). While our in vitro binding data do not exclude formation of Ror $1 / 2$ and Fzd homodimers in the presence of Wnt5a, our findings that Ror2 Ser/Thr phosphorylation requires endogenous Fzd and is not triggered by forced antibody-induced dimerization strongly argue that Ror $1 / 2$ activation depends on Wnt5a-induced clustering of Ror $1 / 2$ and Fzd. Further studies will be needed to determine the stoichiometry of Ror2Wnt5a-Fzd and LRP6-Wnt3a-Fzd tripartite complexes, and the structural determinants responsible for the specific coreceptor-binding properties of different Wnts.

Dvl, a core component of both canonical and noncanonical Wnt signaling, possesses N-terminal DIX, central PDZ, and C-terminal DEP domains (Wallingford and Habas 2005). Whereas DIX and PDZ are required for canonical Wnt signaling, the PDZ and DEP domains have

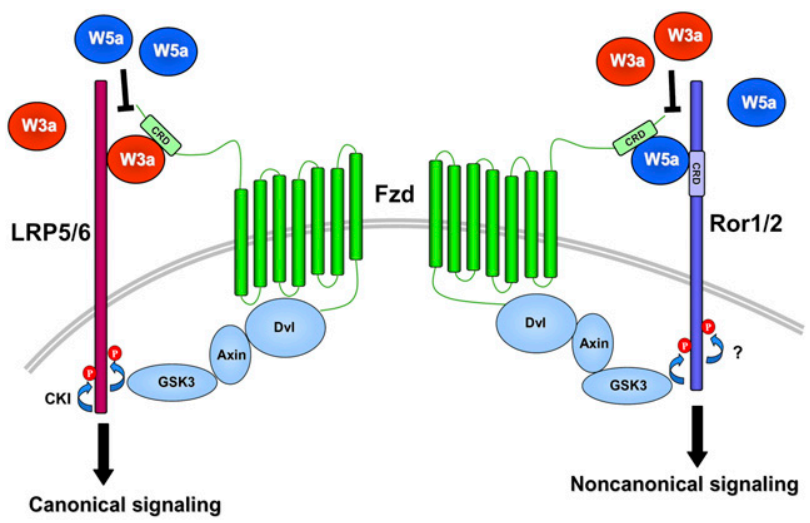

Figure 8. Model for receptor activation by canonical and noncanonical Wnt ligands. See the text for details. 
been shown to participate in noncanonical pathways (Wallingford and Habas 2005). These results have suggested that Dvl acts as a molecular switch to activate canonical or noncanonical signaling (Wallingford and Habas 2005). Our present findings that Dvl knockdown inhibited the phosphorylation of Ror2 as well as that of LRP6 (Bilic et al. 2007; Zeng et al. 2008) imply that this scaffold protein plays an important role in the activation of both classes of Wnt coreceptors. Moreover, a Dvl construct containing only the PDZ and DEP domains served as a dominant negative for Wnt-triggered phosphorylation of both Ror2 and LRP6 (Supplemental Fig. 4B-D). While the DIX domain is known to be involved in Wnt3ainduced phosphorylation of LRP6 (Zeng et al. 2008; Metcalfe et al. 2010), these results argue that this domain also plays an important role in the triggering of noncanonical coreceptors. Of note, Dvl has been shown to activate $\beta$-catenin signaling in the absence of LRP5/6, while expression of the Dvl DEP domain alone stimulated the noncanonical pathway (Wallingford and Habas 2005; Metcalfe et al. 2010). In light of our present findings, it is tempting to speculate that Dvl plays a dual role in both canonical and noncanonical signaling: one as a common component of the shared machinery involved in coreceptor activation, and another downstream and specific for each pathway.

Axin is a crucial component of the $\beta$-catenin destruction complex (Clevers 2006; MacDonald et al. 2009). This protein also acts as a scaffold protein required for GSK3 phosphorylation of LRP5/6 (Zeng et al. 2008). Our studies provide the first demonstration that axin is required for activation of noncanonical Ror2 as well, and that Wnt5a induces the formation of a complex containing endogenous Ror2 and axin. These findings suggest that, following Wnt3a and Wnt5s triggering of Fzd, Dvl and axin allow the recruitment of GSK3 into proximity with LRP5/6 or Ror1/2, respectively, to induce their phosphorylation (Fig. 8). This mechanism is consistent with the "initiation and amplification" model proposed for LRP5/6 activation (Baig-Lewis et al. 2007; Zeng et al. 2008; MacDonald et al. 2009) and would generalize it to noncanonical signaling. Following specific coreceptor activation, the two pathways likely diverge such that, in canonical Wnt signaling, the phosphorylated intracellular domain of LRP5/6 binds to axin and inhibits GSK3 phosphorylation of $\beta$-catenin, resulting in its stabilization (MacDonald et al. 2009). In the Wnt5a noncanonical pathway, the phosphorylated cytoplasmic tails of Ror1 and Ror2 may act as docking sites for other, yet-to-be identified, cytoplasmic effectors.

The Ser/Thr kinase GSK3 is involved in a variety of cellular processes and signaling pathways (Kockeritz et al. 2006). While GSK3 has been reported to participate in Ror2 phosphorylation (Yamamoto et al. 2007), this study provided no evidence for mechanistic or biological implications. Using a phospho-specific antibody generated by us, we identified Ror2 Ser 864 as a GSK3 site phosphorylated in response to Wnt5a. Ser 864 most likely represents the noncanonical equivalent of the GSK3priming phosphorylation sites in LRP6 required for fur- ther phosphorylation and activation of this canonical coreceptor (Zeng et al. 2005). Indeed, we demonstrated that Ser 864 inactivating and phosphomimetic substitutions, respectively, abolished and enhanced the effects of Ror2 on cell motility. Together, these findings provide the first demonstration that Wnt5a-induced Ser phosphorylation is functionally required for activation of Ror biological function.

The role of the Rorl/2 Tyr kinase domain in noncanonical Wnt signaling is controversial /Green et al. 2008). High, nonphysiological levels of Wnt5a were required to induce Ror2 phosphorylation on Tyr, but not Ser/ Thr, residues (Liu et al. 2008), while others (Yamamoto et al. 2007) reported that Wnt5a concentrations sufficient to trigger Ror2 Ser/Thr phosphorylation did not promote Tyr phosphorylation. We showed that Wnt5a induced Ser/Thr, but not Tyr, phosphorylation of Ror2 under conditions in which this receptor exhibited biological function. Conversely, forced Ror2 dimerization by an antibody resulted in Tyr phosphorylation in the absence of Ser/Thr phosphorylation. Furthermore, we showed that wild-type and kinase-dead Ror2 acted similarly upon Wnt5a treatment in both biochemical and functional experiments. These findings as well as other evidence presented here and elsewhere (Nishita et al. 2006; Yamamoto et al. 2007) argue strongly that Wnt5a does not trigger endogenous Ror by the typical mechanism of RTK dimerization to activate noncanonical signaling, but instead couples completely unrelated Ror and Fzd coreceptors.

The fact that the Ror1/2 Tyr kinase domain has been conserved throughout evolution implies its likely biological role. One possibility is that Ror $1 / 2$ has functions independent of any triggered by Wnts. Alternatively, it is possible that, while not directly required for ligand-induced receptor activation, the Ror $1 / 2$ Tyr kinase domain may be involved in the phosphorylation of downstream effectors in certain aspects of its signaling. In this regard, it has been reported that a kinase-dead Ror2 construct was unable to rescue the decreased expression of paraxial protocadherin induced by Ror 2 morpholinos in Xenopus embryos (Schambony and Wedlich 2007).

While it is well established that Wnt5a can inhibit the canonical Wnt pathway, the mechanism involved is controversial, with studies reporting effects either downstream from or upstream of $\beta$-catenin (Ishitani et al. 2003; Topol et al. 2003; Westfall et al. 2003; Mikels and Nusse 2006; Mikels et al. 2009; Sato et al. 2010). We found that Wnt5a and Wnt3a cross-competed for Fzd binding in vitro, and that Wnt5a antagonized Wnt3a signaling in the absence of a requirement for endogenous Ror1/2, consistent with cell surface Wnt ligand competition for Fzd binding (Sato et al. 2010). Moreover, our present findings that Wnt3a similarly inhibits the phosphorylation of Ror2 induced by Wnt5a provides strong evidence that competition at the level of Fzd binding is involved in reciprocal regulation of canonical and noncanonical pathways. Finally, we showed that Wnt3a inhibited hMSC osteogenic differentiation independently of a requirement for $\beta$-catenin activation, implying that canonical Wnt ligands can antagonize the Ror2 pathway at the cell 
surface, and that this effect has biological relevance in cell fate specification. Wnt canonical signaling has important functions in normal bone development (Grigoryan et al. 2008; Liu et al. 2009), and our findings suggest that canonical ligands may act in part by inhibiting the Ror2 pathway, whose role in bone development is strongly supported by analysis of knockout mice as well as identification of Ror2 mutations in human skeletal syndromes (Green et al. 2008).

Other signaling pathways, such as TGF- $\beta /$ BMP (Feng and Derynck 2005), share common as well as distinct coreceptors, but, to our knowledge, none show the unique strategy identified here involving use of the same intracellular components to activate completely unrelated coreceptors. Given the large number of Wnts and the existence of various receptors containing Wnt-binding domains (Green et al. 2008; Angers and Moon 2009), as well as an increasing array of putative noncanonical pathways (Semenov et al. 2007), the common mechanism of coreceptor activation delineated here may represent a general paradigm underlying the activation of other, yet-to-be characterized, Wnt pathways. In this regard, we found that, unlike Wnt5a, other noncanonical Wnts such as Wnt4 and Wnt11 were unable to trigger Ror1-LRP6 or Ror2-LRP6 fusion receptors (L Grumolato and SA Aaronson, unpubl.), suggesting that these ligands may signal through other noncanonical coreceptor(s). Of note, it has been reported recently that Wnt11 functions through Dvl and the Musk receptor during the establishment of zebrafish neuromuscular junctions (Jing et al. 2009). In our model (Fig. 8), the combinatorial nature of Wnt ligand/coreceptor interactions, together with a shared activation mechanism involving a limited number of transduction molecules, allows specificity of signaling through the diversity of ligands, while preserving the principle of economy in receptor activation.

\section{Materials and methods}

\section{Cell culture, transfection, and lentivirus production}

Cell lines were maintained in Dulbecco's modified Eagle's medium (Lonza) supplemented with $10 \%$ fetal bovine serum (Sigma). Control, Wnt3a, and Wnt5a conditioned media (CM) were collected from L cells (American Type Culture Collection [ATCC]) grown according to ATCC instructions. Purified Wnt3a and Wnt5a were purchased from R\&D Systems. SB216763 was purchased form Cayman Chemical. BIO was purchased from Sigma. Control and Dkk1 CM were generated by transient transfection of $293 \mathrm{~T}$ cells with pcDNA3 or Dkk1 (pcDNA3) and collection of the media $2 \mathrm{~d}$ and $3 \mathrm{~d}$ after transfection. Ror $2^{-/-}$ MEFs were derived from mice containing a truncation of Ror2 induced by the insertion of a lacZ cassette after the transmembrane domain that behave as null mutants (DeChiara et al. 2000). We showed previously that PAl ovarian cancer cells exhibit autocrine activation of Wnt signaling (Bafico et al. 2004), while AGS gastric cancer cells contain a $\beta$-catenin-activating mutation (Caca et al. 1999). Primary hMSC cultures (Lonza) were expanded in basal medium according to the manufacturer's instructions or were differentiated using osteogenic medium (Lonza) diluted 1:3 in normal medium. Alkaline phosphatase activity was detected histochemically with the Leukocyte
Alkaline Phosphatase kit (Sigma-Aldrich) or quantified with a luminometer using a chemiluminescent substrate (BioFX Laboratories) and normalized to total proteins.

Transient transfection was performed using Fugene 6 (Roche) according to the manufacturer's instructions or with polyethylenimine (Polysciences). For lentivirus production, 293T cells were cotransfected with the lentiviral vector and pCMV $\Delta 8.91$ and pMD VSV-G plasmids (kindly provided by I. Weissman). Two days after transfection, CM was collected, supplemented with $8 \mu \mathrm{g} / \mathrm{mL}$ polybrene, and added for overnight incubation to freshly plated cells to be transduced. Two days after infection, the cells were selected in $2 \mu \mathrm{g} / \mathrm{mL}$ puromycin or $10 \mu \mathrm{g} / \mathrm{mL}$ blasticidin.

siRNAs (Dharmacon) were used to knock down the three predominant Fzd coreceptors in 293T cells-i.e., Fzd2, Fzd4, and Fzd5-as reported previously (Pan et al. 2008). 293T cells were transfected using Lipofectamine 2000 (Invitrogen) according to the manufacturer's instructions, and $2 \mathrm{~d}$ later the cells were treated with CM or purified Wnt3a and Wnt5a (R\&D Systems). As a control, an siRNA targeting GFP was used.

\section{Constructs}

The DKK2C-Wnt5a chimera was generated by fusion of the LRPbinding domain of mouse Dkk2 (amino acids 141-259) and Wnt5a (Liu et al. 2005). The Ror2-LRP6 and Ror1-LRP6 constructs (pcDNA3) were generated by fusion of the human Ror2 or Rorl extracellular domains and human LRP6 transmembrane and intracellular domains. Human Dkk1 was cloned in pcDNA3-Flag. The $\beta$-catenin/TCF reporter Super8XTOPFlash, containing eight TCF-responsive elements driving the firefly luciferase gene, was kindly provided by R. Moon. The LRP6 extracellular domain fused to the IgG heavy chain (extLRP6-Fc) was cloned in pcDNA3 vector, and the CRD of Fzd8 was cloned in pcDNA3-Flag (Fzd8-CRD-Flag). All lentiviral shRNA constructs were generated in VIRHD-EP (kindly provided by $\mathrm{L}$. Gusella) containing either puromycin or blasticidin resistance genes, mostly using target sequences from the literature (sequences available on request). Other constructs generated using this lentiviral vector include the extracellular domain of human Ror2 (amino acids 1-394) with an HA tag at the C terminus (extRor2HA); a dominant-negative Dvl (including the PDZ and DEP domains of human Dvll) fused to GFP at the $\mathrm{N}$ terminus; human wild-type Ror2; Ror2-S864A, Ror2-K507A, Ror2-S864D, and Ror2-S864E with an N-terminal Flag tag; dominant-negative TCF4 fused to GFP; and Shisa, cloned by reverse transcription and PCR from Xenopus embryos and containing a myc tag at the C terminus. GSK-3 putative phosphorylation sites in the Ror2 intracellular domain were identified using the Scansite motif Scanner, and Ror2 mutant constructs were generated using the QuikChange II Site-Directed Mutagenesis kit (Stratagene) with $\mathrm{N}$-terminal Flag-tagged Ror2 constructs (pCS2 vector or VIRHDEP lentiviral vector) as templates. The lentiviral $\beta$-catenin/TCF luciferase reporter containing 14 wild-type (MegaTop-LentiLuc) or mutant (MegaFop-LentiLuc) TCF consensus sequences was generated by PCR from a seven-repeat-containing construct described previously (Akiri et al. 2009). All constructs were sequence-verified.

\section{Antibodies}

The anti-LRP5 mouse monoclonal antibody was generated following the procedure previously used for the anti-LRP6 antibody (Khan et al. 2007). Nonphospho-Ror2 antibody was generated by Proteintech using the peptide VPKPSSHHS ${ }^{\star}$ GSGSTS (asterisk indicates phosphorylated residue) conjugated to keyhole limpet hemocyanin. The bleeds were purified on a nonphosphopeptide 
column using the CarboxyLink Immobilization kit (Thermo Fisher Scientific), and the specificity of the antibody was assessed by slot blot using serial dilutions of the nonphosphopeptide and phosphopeptide. Rabbit anti-LRP6, rabbit anti-phospho-LRP6 (S1490), rabbit anti-Wnt5a, rabbit anti-phospho-Thr (Cell Signaling Technology), rabbit anti-Dvl2, mouse anti-Dvl3 (Santa Cruz Biotechnology), mouse anti-Flag, mouse anti-tubulin (Sigma), rabbit anti-phospho-Ser (Invitrogen), goat anti-Ror2, goat antiRor1, rat anti-Wnt3a, goat anti-axin (R\&D Systems), mouse antiphospho-Tyr clone 4G10 (Millipore), and mouse anti- $\beta$-catenin (BD Biosciences) were from commercial sources. Mouse antimyc clone 9E10 was obtained from the Mount Sinai Hybridoma Core Facility.

\section{Free $\beta$-catenin, $\mathrm{PIP}_{2}$ quantification, and luciferase assays}

Uncomplexed $\beta$-catenin was measured by the GST-E-cadherinbinding assay as described previously (Liu et al. 2003). The amounts of $\mathrm{PIP}_{2}$ were measured by ELISA as described previously (Pan et al. 2008). For luciferase assay, cells transfected or transduced with the $\beta$-catenin/TCF firefly luciferase reporters and the renilla luciferase control reporters were lysed, and luciferase activity was measured using the Dual-Luciferase Reporter Assay System (Promega) according to the manufacturer's instructions.

\section{In vitro binding assay}

CM was collected from 293 cells stably expressing extLRP6-Fc; L cells stably expressing extRor2-HA, Wnt3a, or Wnt5a; and 293T cells transiently transfected with Fzd8-CRD-Flag. ExtLRP6-Fc, Fzd8-CRD-Flag, and extRor2-HA CM were purified overnight with anti-Fc (Sigma), anti-Flag (Sigma), or anti-HA (Covance) beads, respectively. The beads were then washed and incubated with equal volumes of Wnt CM. After washing, the beads were incubated overnight with CM containing the other coreceptor and washed. Loading buffer was then added to the beads, and SDS-PAGE was performed, followed by immunoblot analysis.

\section{Wound healing assay}

Confluent rat1 cells and MEFs plated on fibronectin-coated (Sigma) wells were maintained for $1 \mathrm{~d}$ (rat1) or overnight in $0.5 \%$ FBS medium. The cells were then scratched with a sterile $1-\mathrm{mL}$ tip, washed twice in PBS, and incubated with control or Wnt5a medium containing $0.5 \%$ FBS. Photographs of the cells were taken at time 0 and $15 \mathrm{~h}$ (rat1) or $8 \mathrm{~h}$ (MEFs) after scratch using an inverted microscope. The relative distance was calculated using ImageJ (http://rsb.info.nih.gov/ij/index.html).

\section{Acknowledgments}

We thank L. Gusella, I. Weissman, R. Moon, A. Yamamoto, A. Yaniv, A. Gazit, T. Villanueva de la Torre, Z. Khan, B. Zhao, S.K.A. Divakar, G. Akiri, and S. Asciutti for providing reagents. We also thank M. Mlodzik, S. Sokol, H. Hikasa, J. Chen, L. Chen, M. Swan, J. Lee, R. Diotti, C. Munoz-Fontela, and S.K. Mungamuri for helpful comments and discussion. L.G. is recipient of a DOD Prostate Cancer Post-doctoral Fellowship (PC050673) and a New York State Department of Health Breast Cancer Research Postdoctoral Fellowship Award. G.L. received support from the Leo and Julia Forchheimer Foundation and Breast Cancer Alliance, Inc. S.V. received a Post-doctoral Fellowship from the American Urological Association Foundation. This study was supported by grants from the Breast Cancer Research Foundation, the Leo and Julia Forchheimer Foundation, and the National Cancer Institute (CA071672 and T32CA078207).

\section{References}

Akiri G, Cherian MM, Vijayakumar S, Liu G, Bafico A, Aaronson SA. 2009. Wnt pathway aberrations including autocrine Wnt activation occur at high frequency in human non-small-cell lung carcinoma. Oncogene 28: 2163-2172.

Angers S, Moon RT. 2009. Proximal events in Wnt signal transduction. Nat Rev Mol Cell Biol 10: 468-477.

Bafico A, Gazit A, Pramila T, Finch PW, Yaniv A, Aaronson SA. 1999. Interaction of frizzled related protein (FRP) with Wnt ligands and the frizzled receptor suggests alternative mechanisms for FRP inhibition of Wnt signaling. J Biol Chem 274: 16180-16187.

Bafico A, Liu G, Goldin L, Harris V, Aaronson SA. 2004. An autocrine mechanism for constitutive Wnt pathway activation in human cancer cells. Cancer Cell 6: 497-506.

Baig-Lewis S, Peterson-Nedry W, Wehrli M. 2007. Wingless/Wnt signal transduction requires distinct initiation and amplification steps that both depend on Arrow/LRP. Dev Biol 306: 94-111.

Bilic J, Huang YL, Davidson G, Zimmermann T, Cruciat CM, Bienz M, Niehrs C. 2007. Wnt induces LRP6 signalosomes and promotes dishevelled-dependent LRP6 phosphorylation. Science 316: 1619-1622.

Billiard J, Way DS, Seestaller-Wehr LM, Moran RA, Mangine A, Bodine PV. 2005. The orphan receptor tyrosine kinase Ror2 modulates canonical Wnt signaling in osteoblastic cells. Mol Endocrinol 19: 90-101.

Caca K, Kolligs FT, Ji X, Hayes M, Qian J, Yahanda A, Rimm DL, Costa J, Fearon ER. 1999. $\beta$ - and $\gamma$-catenin mutations, but not E-cadherin inactivation, underlie T-cell factor/lymphoid enhancer factor transcriptional deregulation in gastric and pancreatic cancer. Cell Growth Differ 10: 369-376.

Clevers H. 2006. Wnt/ $\beta$-catenin signaling in development and disease. Cell 127: 469-480.

Dann CE, Hsieh JC, Rattner A, Sharma D, Nathans J, Leahy DJ. 2001. Insights into Wnt binding and signalling from the structures of two Frizzled cysteine-rich domains. Nature 412: $86-90$.

Davidson G, Wu W, Shen J, Bilic J, Fenger U, Stannek P, Glinka A, Niehrs C. 2005. Casein kinase $1 \gamma$ couples Wnt receptor activation to cytoplasmic signal transduction. Nature 438: 867-872.

DeChiara TM, Kimble RB, Poueymirou WT, Rojas J, Masiakowski P, Valenzuela DM, Yancopoulos GD. 2000. Ror2, encoding a receptor-like tyrosine kinase, is required for cartilage and growth plate development. Nat Genet 24: 271-274.

Feng XH, Derynck R. 2005. Specificity and versatility in TGF- $\beta$ signaling through Smads. Annu Rev Cell Dev Biol 21: 659-693.

Fukuda T, Chen L, Endo T, Tang L, Lu D, Castro JE, Widhopf GF II, Rassenti LZ, Cantwell MJ, Prussak CE, et al. 2008. Antisera induced by infusions of autologous Ad-CD154leukemia B cells identify ROR1 as an oncofetal antigen and receptor for Wnt5a. Proc Natl Acad Sci 105: 3047-3052.

Gonzalez-Sancho JM, Brennan KR, Castelo-Soccio LA, Brown AM. 2004. Wnt proteins induce dishevelled phosphorylation via an LRP5/6- independent mechanism, irrespective of their ability to stabilize $\beta$-catenin. Mol Cell Biol 24: 4757-4768.

Green JL, Kuntz SG, Sternberg PW. 2008. Ror receptor tyrosine kinases: Orphans no more. Trends Cell Biol 18: 536-544.

Grigoryan T, Wend P, Klaus A, Birchmeier W. 2008. Deciphering the function of canonical Wnt signals in development and disease: Conditional loss- and gain-of-function mutations of $\beta$-catenin in mice. Genes Dev 22: 2308-2341.

He X, Saint-Jeannet JP, Wang Y, Nathans J, Dawid I, Varmus H. 1997. A member of the Frizzled protein family mediating axis induction by Wnt-5A. Science 275: 1652-1654. 
Hikasa H, Shibata M, Hiratani I, Taira M. 2002. The Xenopus receptor tyrosine kinase Xror2 modulates morphogenetic movements of the axial mesoderm and neuroectoderm via Wnt signaling. Development 129: 5227-5239.

Ishitani T, Kishida S, Hyodo-Miura J, Ueno N, Yasuda J, Waterman M, Shibuya H, Moon RT, Ninomiya-Tsuji J, Matsumoto K. 2003. The TAK1-NLK mitogen-activated protein kinase cascade functions in the Wnt-5a/Ca ${ }^{2+}$ pathway to antagonize Wnt/ $\beta$-catenin signaling. Mol Cell Biol 23: 131-139.

Jing L, Lefebvre JL, Gordon LR, Granato M. 2009. Wnt signals organize synaptic prepattern and axon guidance through the zebrafish unplugged/MuSK receptor. Neuron 61: 721-733.

Khan Z, Vijayakumar S, de la Torre TV, Rotolo S, Bafico A. 2007. Analysis of endogenous LRP6 function reveals a novel feedback mechanism by which Wnt negatively regulates its receptor. Mol Cell Biol 27: 7291-7301.

Kikuchi A, Yamamoto H, Sato A. 2009. Selective activation mechanisms of Wnt signaling pathways. Trends Cell Biol 19: 119-129.

Kockeritz L, Doble B, Patel S, Woodgett JR. 2006. Glycogen synthase kinase-3-An overview of an over-achieving protein kinase. Curr Drug Targets 7: 1377-1388.

Liu G, Bafico A, Harris VK, Aaronson SA. 2003. A novel mechanism for Wnt activation of canonical signaling through the LRP6 receptor. Mol Cell Biol 23: 5825-5835.

Liu G, Bafico A, Aaronson SA. 2005. The mechanism of endogenous receptor activation functionally distinguishes prototype canonical and noncanonical Wnts. Mol Cell Biol 25: 3475-3482.

Liu Y, Bhat RA, Seestaller-Wehr LM, Fukayama S, Mangine A, Moran RA, Komm BS, Bodine PV, Billiard J. 2007. The orphan receptor tyrosine kinase Ror2 promotes osteoblast differentiation and enhances ex vivo bone formation. Mol Endocrinol 21: 376-387.

Liu Y, Rubin B, Bodine PV, Billiard J. 2008. Wnt5a induces homodimerization and activation of Ror2 receptor tyrosine kinase. J Cell Biochem 105: 497-502.

Liu G, Vijayakumar S, Grumolato L, Arroyave R, Qiao H, Akiri G, Aaronson SA. 2009. Canonical Wnts function as potent regulators of osteogenesis by human mesenchymal stem cells. J Cell Biol 185: 67-75.

Logan CY, Nusse R. 2004. The Wnt signaling pathway in development and disease. Annu Rev Cell Dev Biol 20: 781810.

MacDonald BT, Tamai K, He X. 2009. Wnt/ $\beta$-catenin signaling: Components, mechanisms, and diseases. Dev Cell 17: 9-26.

Metcalfe C, Mendoza-Topaz C, Mieszczanek J, Bienz M. 2010. Stability elements in the LRP6 cytoplasmic tail confer efficient signalling upon DIX-dependent polymerization. J Cell Sci 123: 1588-1599.

Mikels AJ, Nusse R. 2006. Purified Wnt5a protein activates or inhibits $\beta$-catenin-TCF signaling depending on receptor context. PLoS Biol 4: e115. doi: 10.1371/journal.pbio.0040115.

Mikels A, Minami Y, Nusse R. 2009. Ror2 receptor requires tyrosine kinase activity to mediate Wnt5A signaling. I Biol Chem 284: 30167-30176.

Nishita M, Yoo SK, Nomachi A, Kani S, Sougawa N, Ohta Y, Takada S, Kikuchi A, Minami Y. 2006. Filopodia formation mediated by receptor tyrosine kinase Ror2 is required for Wnt5a-induced cell migration. J Cell Biol 175: 555-562.

Nishita M, Enomoto M, Yamagata K, Minami Y. 2010a. Cell/ tissue-tropic functions of Wnt5a signaling in normal and cancer cells. Trends Cell Biol 20: 346-354.

Nishita M, Itsukushima S, Nomachi A, Endo M, Wang Z, Inaba D, Qiao S, Takada S, Kikuchi A, Minami Y. 2010b. Ror2/
Frizzled complex mediates Wnt5a-induced AP-1 activation by regulating Dishevelled polymerization. Mol Cell Biol 30: 3610-3619.

Nomachi A, Nishita M, Inaba D, Enomoto M, Hamasaki M, Minami Y. 2008. Receptor tyrosine kinase Ror2 mediates Wnt5a-induced polarized cell migration by activating c-Jun N-terminal kinase via actin-binding protein filamin A. J Biol Chem 283: 27973-27981.

Oishi I, Suzuki H, Onishi N, Takada R, Kani S, Ohkawara B, Koshida I, Suzuki K, Yamada G, Schwabe GC, et al. 2003. The receptor tyrosine kinase Ror2 is involved in noncanonical Wnt5a/JNK signalling pathway. Genes Cells 8: 645-654.

Pan W, Choi SC, Wang H, Qin Y, Volpicelli-Daley L, Swan L, Lucast L, Khoo C, Zhang X, Li L, et al. 2008. Wnt3a-mediated formation of phosphatidylinositol 4,5-bisphosphate regulates LRP6 phosphorylation. Science 321: 1350-1353.

Pittenger MF, Mackay AM, Beck SC, Jaiswal RK, Douglas R, Mosca JD, Moorman MA, Simonetti DW, Craig S, Marshak DR. 1999. Multilineage potential of adult human mesenchymal stem cells. Science 284: 143-147.

Qian D, Jones C, Rzadzinska A, Mark S, Zhang X, Steel KP, Dai X, Chen P. 2007. Wnt5a functions in planar cell polarity regulation in mice. Dev Biol 306: 121-133.

Sato A, Yamamoto H, Sakane H, Koyama H, Kikuchi A. 2010. Wnt5a regulates distinct signalling pathways by binding to Frizzled2. EMBO J 29: 41-54.

Schambony A, Wedlich D. 2007. Wnt-5A/Ror2 regulate expression of XPAPC through an alternative noncanonical signaling pathway. Dev Cell 12: 779-792.

Schlessinger J. 2000. Cell signaling by receptor tyrosine kinases. Cell 103: 211-225.

Schlessinger K, McManus EJ, Hall A. 2007. Cdc42 and noncanonical Wnt signal transduction pathways cooperate to promote cell polarity. J Cell Biol 178: 355-361.

Semenov MV, Habas R, Macdonald BT, He X. 2007. SnapShot: Noncanonical Wnt signaling pathways. Cell 131: 1378.e11378.e2. doi: 10.1016/j.cell.2007.12.011.

Simons M, Mlodzik M. 2008. Planar cell polarity signaling: From fly development to human disease. Annu Rev Genet 42: 517-540.

Tamai K, Semenov M, Kato Y, Spokony R, Liu C, Katsuyama Y, Hess F, Saint-Jeannet JP, He X. 2000. LDL-receptor-related proteins in Wnt signal transduction. Nature 407: 530-535.

Topol L, Jiang X, Choi H, Garrett-Beal L, Carolan PJ, Yang Y. 2003. Wnt-5a inhibits the canonical Wnt pathway by promoting GSK-3-independent $\beta$-catenin degradation. I Cell Biol 162: 899-908.

van Amerongen R, Mikels A, Nusse R. 2008. Alternative Wnt signaling is initiated by distinct receptors. Sci Signal 1: re9. doi: 10.1126/scisignal.135re9.

Wallingford JB, Habas R. 2005. The developmental biology of Dishevelled: An enigmatic protein governing cell fate and cell polarity. Development 132: 4421-4436.

Wang Y, Guo N, Nathans J. 2006. The role of Frizzled3 and Frizzled6 in neural tube closure and in the planar polarity of inner-ear sensory hair cells. J Neurosci 26: 2147-2156.

Westfall TA, Brimeyer R, Twedt J, Gladon J, Olberding A, Furutani-Seiki M, Slusarski DC. 2003. Wnt-5/pipetail functions in vertebrate axis formation as a negative regulator of Wnt/ $\beta$-catenin activity. J Cell Biol 162: 889-898.

Yamaguchi TP, Bradley A, McMahon AP, Jones S. 1999. A Wnt5a pathway underlies outgrowth of multiple structures in the vertebrate embryo. Development 126: 1211-1223.

Yamamoto A, Nagano T, Takehara S, Hibi M, Aizawa S. 2005. Shisa promotes head formation through the inhibition of 
Grumolato et al.

receptor protein maturation for the caudalizing factors, Wnt and FGF. Cell 120: 223-235.

Yamamoto H, Yoo SK, Nishita M, Kikuchi A, Minami Y. 2007. Wnt5a modulates glycogen synthase kinase 3 to induce phosphorylation of receptor tyrosine kinase Ror2. Genes Cells 12: 1215-1223.

Yamamoto S, Nishimura O, Misaki K, Nishita M, Minami $\mathrm{Y}_{,}$ Yonemura S, Tarui H, Sasaki H. 2008. Cthrcl selectively activates the planar cell polarity pathway of Wnt signaling by stabilizing the Wnt-receptor complex. Dev Cell 15: 23 36.

Zeng X, Tamai K, Doble B, Li S, Huang H, Habas R, Okamura H, Woodgett J, He X. 2005. A dual-kinase mechanism for Wnt co-receptor phosphorylation and activation. Nature 438: 873-877.

Zeng X, Huang H, Tamai K, Zhang X, Harada Y, Yokota C, Almeida K, Wang J, Doble B, Woodgett J, et al. 2008. Initiation of Wnt signaling: Control of Wnt coreceptor Lrp6 phosphorylation/activation via frizzled, dishevelled and axin functions. Development 135: 367-375. 


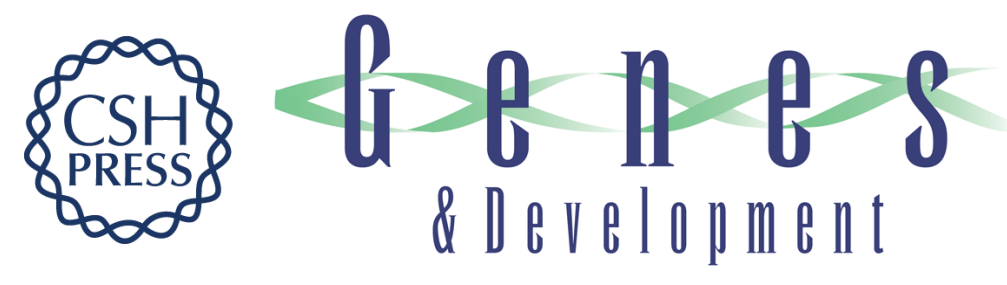

\section{Canonical and noncanonical Wnts use a common mechanism to activate completely unrelated coreceptors}

Luca Grumolato, Guizhong Liu, Phyllus Mong, et al.

Genes Dev. 2010, 24:

Access the most recent version at doi:10.1101/gad.1957710

\section{Supplemental http://genesdev.cshlp.org/content/suppl/2010/11/03/24.22.2517.DC1 Material}

Related Content

Shared Means to Distinct Ends

Annalisa M. VanHook

Sci. Signal. November , 2010 3: ec356

References This article cites 60 articles, 27 of which can be accessed free at:

http://genesdev.cshlp.org/content/24/22/2517.full.html\#ref-list-1

Articles cited in:

http://genesdev.cshlp.org/content/24/22/2517.full.html\#related-urls

\section{License}

Email Alerting Receive free email alerts when new articles cite this article - sign up in the box at the top Service right corner of the article or click here.

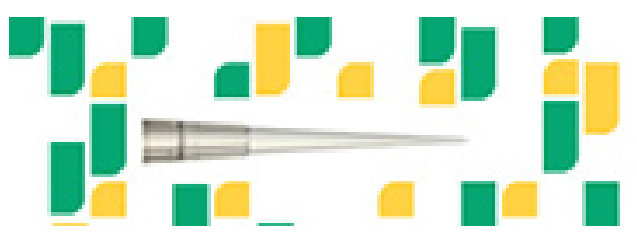

Focused on your science. 